\title{
Editors’ Biographical Sketches
}

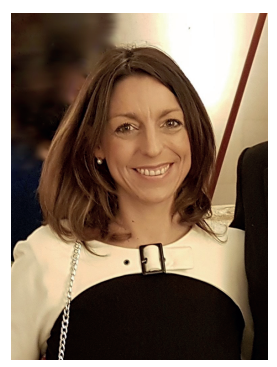

Evelina Colacino received her double PhD (with European Label) in 2002 at the University of Montpellier II, France, and at the University of Calabria, Italy. She was appointed as research fellow at the Catholic University of Louvain (Belgium, 2003), working on the preparation of new hydantoin scaffolds as antibacterial agents, research scientist at Sigma-Tau Pharmaceuticals (Italy, 2004) and postdoctoral fellow at the University of Montpellier II (France, until 2007). She was hired as assistant professor in 2008 and associate professor of organic and green chemistry, since 2013, at the University of Montpellier, France. Her main research activities concern the development of eco-friendly methodologies for the preparation of biomolecules, heterocyclic compounds and hybrid materials by mechanochemistry (dry or wet grinding), with main focus on hydantoin scaffold and active pharmaceutical ingredients (medicinal mechanochemistry). She also investigates sustainable approaches to homogeneous or heterogeneous metal-catalyzed processes by combining enabling technologies (ultrasounds, microwaves and flow systems) with nonconventional media (e.g., glycerol, water, poly(ethylene)glycols and PEG-based ionic liquids) or in micellar conditions (in water and glycerol). She has (co)authored five book chapters, two patents and 70 peer-reviewed scientific publications.

She is a member of the American Chemical Society, the International Mechanochemical Association and Beyond Benign association for green chemistry education. She is a promoter of sustainability in higher education by integrating green chemistry at undergraduate level in organic chemistry courses, teaching laboratories and across the subdisciplines of chemistry, with a special focus on the fundamentals and the practice of mechanochemistry.

Since 2019, she leads the European Programme COST Action CA18112 (MechSustInd, 2019-2023) - 'Mechanochemistry for Sustainable Industry (www.mechsustind.eu and https:// www.cost.eu).

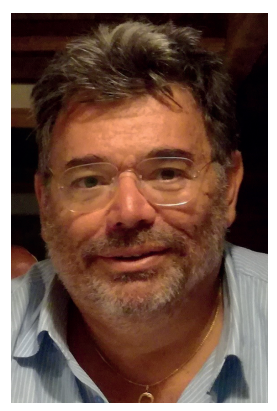

Guido Ennas, graduated in chemistry at University of Cagliari (1986), received his master's degree in materials science at the Polytechnic of Turin (1988), and his PhD in chemistry at the University of Cagliari (1991). He worked as lecturer at the University of Cagliari from 1992 to 2001. Since 2001, he has been associate professor of inorganic chemistry at the Pharmacy Faculty of the University of Cagliari. His roles have included coordinator of the School of Pharmacy at the University of Cagliari and member of the Executive Board of the Consortium INSTM (National Interuniversity Consortium of Materials Science and Technology). Prof. Ennas is responsible of the Micro- and Nanostructured Materials Research Group, having more than 20-year research experience in design, synthesis and characterization of nanostructured, micro- and mesoporous materials, with particular relevance to the development of different inorganic materials and coordination polymers by mechanochemical approach. He has published more than 100 scientific articles and two national patents and presented his results at national and international conferences. He is reviewer for several international journals in the field of materials science. 


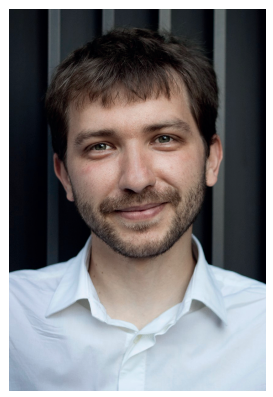

Ivan Halasz graduated in chemistry in 2003 and obtained his PhD with Prof. Hrvoj Vančik in 2008 from the University of Zagreb. Following a twoyear postdoctorate with Prof. Robert Dinnebier at the Max-Planck-Institute for solid-state research in Stuttgart, he joined as a faculty at the Ruđer Bošković Institute in Zagreb in 2012, where he holds the position of a senior researcher (equivalent to an associate professor) since 2016. His research interests are focused on solid state, including solid-state reactivity, mechanochemistry, powder X-ray diffraction, structural characterization and reaction mechanisms. He published around 80 research papers and coauthored two high-school chemistry textbooks. In 2016, he received the Croatian State Science Award for his contributions in developing in situ methods for the study of mechanochemical reactions. He is currently the leader of the working package of the COST Action CA18112 "Mechanochemistry for Sustainable Industry" (MechSustlnd) dedicated to in situ monitoring.

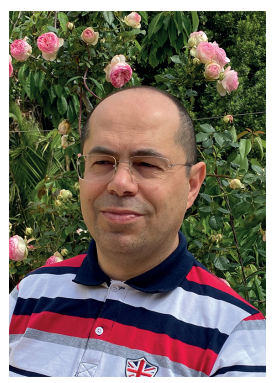

Andrea Porcheddu studied chemistry at the University of Sassari and got his "Laurea" degree in chemistry in 1995 with first-class honors. His diploma thesis dealt with the synthesis of piperazic acid derivatives as CPP analogues, which was carried out under the direction of Dr. Massimo Falorni. He then undertook doctoral research, designing novel strategies to use cyanuric chloride (TCT) in friendly organic processes under the supervision of Prof. Maurizio Taddei at the University of Sassari and was awarded his PhD in 1999. He completed postdoctoral studies (2000) in the group of Prof. Charles Mioskowsky at the Louis Pasteur University (Strasbourg, France) working on the synthesis of quinuclidine derivatives. In 2001, he moved back to Sassari University where he was appointed as assistant professor. In January 2015, he joined the Chemistry Department of the University of Cagliari (Italy), where he currently has a permanent position as associate professor. His diverse experience ranges from the synthesis of chimera molecules possessing complex molecular architecture to environmentally friendlier alternatives for synthesis using the most advanced technologies such as solid-phase synthesis, combinatorial chemistry, green chemistry in eutectic solvents, microwaves and mechanochemical mixing. The second field of interest concerns the development of novel Borrowing Hydrogen and Transfer Hydrogenation strategies for making $\mathrm{C}-\mathrm{N}$ bonds using tertiary amines or alcohols instead of more labile aldehydes. The scientific interests of Prof. Porcheddu are also focused on finding novel and highly efficient nonconventional catalysts (mainly $\mathrm{Fe}$ and $\mathrm{Cu}$ ) and reagents for $\mathrm{C}-\mathrm{H}$ bond activation, intending to minimize both waste production and energy consumption. Currently, his main scientific interest covers the field of ballmilling chemistry, and is devoted to the study of nonconventional transformations over the field of organic chemistry, which can reduce solvents, by-products and wastes, and they can be defined as environmentally sustainable.

Prof. Porcheddu has authored six-chapter books and he is author or coauthor of more than 90 scientific publications on refereed international journals (H index, Scopus $=32$ ).

Presently, he represents Italy in the Management Committee of the European Programme COST Action CA18112 (MechSustInd) - "Mechanochemistry for Sustainable Industry" (MechSustInd). 


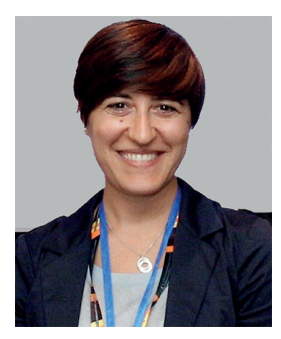

Dr. Alessandra Scano graduated with Master of Science in pharmaceutical chemistry and technology (2005) and in cellular and molecular biology (2019) at the University of Cagliari. In 2009, she received her PhD in chemical science. She spent one year (2009) as postdoctorate at Materials and Surface Science Institute, University of Limerick (Ireland). From 2010 to 2013, she worked as researcher in synthesis (top-down and bottom-up approaches) and characterization of nanomaterials at the Asociacion de la Industria Navarra, Spain. Since 2014, she is back to University of Cagliari where she is part of the Micro- and Nanostructured Materials Research Group. She works on the development of micro- and nanostructured materials for biomedical applications with particular interest on the mechanochemical approach. She is author/coauthor of several papers published in international journals, and she has presented her results at several international conferences. Dr. Scano has also experience in design and management of international and national projects, and she is part of the EUPF (Register of Euro-Projects Designers and Managers - Europe Project Forum Foundation). 
